“C 2016 IEEE. Personal use of this material is permitted. Permission from IEEE must be obtained for all other uses, in any current or future media, including reprinting/republishing this material for advertising or promotional purposes, creating new collective works, for resale or redistribution to servers or lists, or reuse of any copyrighted component of this work in other works." 


\title{
SSRBC 2016: Sclera Segmentation and Recognition Benchmarking Competition
}

\author{
Abhijit Das ${ }^{\mathrm{a}}$, Umapada Pal ${ }^{\mathrm{b}}$, Miguel A. Ferrer ${ }^{\mathrm{c}}$ and Michael Blumenstein ${ }^{\mathrm{d}}$

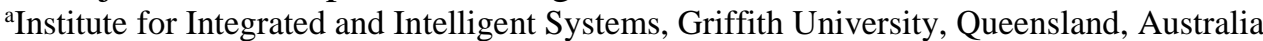 \\ abhijit.dasegriffithuni.edu.au, m.blumenstein@griffith.edu.au \\ ${ }^{\mathrm{b} C}$ Computer Vision and Pattern Recognition Unit, Indian Statistical Institute, Kolkata, India, umapada@isical .ac . in \\ 'IDeTIC, University of Las Palmas de Gran Canaria, Las Palmas, Spain, mferreredsc.ulpgc.es \\ ${ }^{\mathrm{d} S c h o o l ~ o f ~ S o f t w a r e, ~ U n i v e r s i t y ~ o f ~ T e c h n o l o g y ~ S y d n e y, ~ A u s t r a l i a, ~ m i c h a e l . b l u m e n s t e i n @ u t s . e d u . a u ~}$
}

\begin{abstract}
This article reports and summarizes the results of a competition on sclera segmentation and recognition benchmarking, called Sclera Segmentation and Recognition Benchmarking Competition 2016 (SSRBC 2016). It was organized in the context of the 9th IAPR International Conference on Biometrics (ICB 2016). The goal of this competition was to record the recent developments in sclera segmentation and recognition, and also to gain the attention of researchers on this subject of biometrics. In this regard, we have used a multi-angle sclera dataset (MASD version 1). It is comprised of 2624 images taken from both the eyes of 82 identities. Therefore, it consists of images of $164(82 * 2)$ different eyes. We have prepared a manual segmentation mask of these images to create the baseline for both tasks. We have, furthermore, adopted precision and recall based statistical measures to evaluate the effectiveness of the segmentation and the ranks of the competing algorithms. The recognition accuracy measure has been employed to measure the recognition task. To summarize, twelve participants registered for the competition, and among them, three participants submitted their algorithms/ systems for the segmentation task and two their recognition algorithm. The results produced by these algorithms reflect developments in the literature of sclera segmentation and recognition, employing cutting edge segmentation techniques. Along with the algorithms of three competing teams and their results, the MASD version 1 dataset will also be freely available for research purposes from the organizer's website. The competition also demonstrates the recent interests of researchers from academia as well as industry on this subject of biometrics.
\end{abstract}

\section{Introduction}

The white region in the eye, around the eyeball, which contains blood vessel patterns, is known as the sclera. These blood vessel patterns, also termed as conjunctival vasculature, can be employed for personal identification. The very recent literature refers to the success of sclera biometrics among other ocular biometric traits [3-7].

The major reason for the attractiveness of this biometric is due to its applicability. This biometric in conjunction with the iris biometric can enhance the relevance of the iris biometric in non-ideal conditions. As an emerging trait, it is first necessary to assess the biometric usefulness of the sclera independently for larger populations as well as the robustness of the trait under varying conditions. Moreover the research conducted on this subject is very limited and not extensively studied. Furthermore, sclera segmentation and recognition is a significantly important part of sclera biometrics. However, sclera segmentation and recognition has not been extensively investigated as a separate topic, but mainly summarized as a component of a broader task. Therefore, to-date the literature related to sclera segmentation is still in its early stage of investigation and little is known in regard to its challenges.

Various segmentation algorithms for sclera segmentation and recognition are proposed in subsequent works in the literature, but there are a number of unsolved challenges, which make this an open research area. To be specific these independent works on sclera segmentation, which are addressed in the literature, were evaluated employing independent in-house datasets or on public datasets with fewer challenging sclera images. Therefore it will be helpful for future researchers if a common platform is set for the evaluation of sclera segmentation and recognition algorithms. As a result, the $1^{\text {st }}$ Sclera Segmentation Benchmarking Competition (SSBC 2015) was organized in the context of the IEEE Seventh International Conference on Biometrics: Theory, Applications and Systems BTAS 2015. The successful organization and the appreciating impact of this competition have inspired the organizers to plan further competitions on sclera segmentation and recognition namely: SSRBC 2016.

The main aim of the competition is to establish a standard benchmark for sclera recognition with a common dataset and also to record the recent developments of sclera segmentation that took place after SSBC 2015. More importantly this competition will stress the importance of sclera segmentation of multi-angle eye images and eye images in varying illuminations and lighting conditions. Moreover, the additional aim of establishing this competition was also to attract more interest from researchers on this particular subject. Furthermore, another impact of the competition is to come up with a structured and systematic reference document for enduring research on this subject. The conceived competition is adhering to 
biometric research, and this is the reason it is hosted in the context of the 9th IAPR International Conference on Biometrics (ICB 2016); details about the conference are available from: http://www.icb2016.hh.se/Welcome. Details about the competition can be found at: https://sites.google.com/site/ssrb2016/home.

The rest of this paper is organized as follows. In section 2 the competition schedule, the dataset for the competition and the performance evaluation technique adopted to evaluate and rank the participant's algorithm are described. In section 3, various algorithms from the participants are described in details, whereas in section 4, the results achieved from the submitted algorithms and their detailed analysis is summarized. Finally, the last section i.e. section 5 , the overall conclusions are drawn and the future scope of this research is discussed.

\section{The SSRBC 2016 competition}

The competition schedule is shown in table 1 .

Table 1: Schedule of the competition

\begin{tabular}{|c|c|}
\hline Different Phases & Dates \\
\hline Site opens & 1st September, 2015 \\
\hline Registration starts & 1st September, 2015 \\
\hline Test dataset available & 1st September, 2015 \\
\hline Registration closes & 18 th January 2016 \\
\hline Algorithm submission & 18 th January 2016 \\
\hline Results announcement & $19^{\text {th }}$ January 2016 \\
\hline
\end{tabular}

The call for participation in the competition was promoted via the website of the competition and further communications were made via email with the researchers. The competition registrations were performed by email request from the participants with the following information: Name, Affiliation, Email Address, Phone Number and Mailing Address of the participants. Twelve participants registered for the competition from distinguished laboratories and industry, located in different countries. Among them, three participants submitted their algorithms. Table 2 reflects the name and the affiliation of participants those who submitted their algorithms.

Table 2. Descriptions of the participating teams details

\begin{tabular}{|c|c|}
\hline $\begin{array}{c}\text { Participating } \\
\text { teams }\end{array}$ & Name (Institution)/ Task \\
\hline \hline 1 & $\begin{array}{c}\text { Aruna Kumar S V (SJCE, Mysusru, Karnataka, } \\
\text { India) /segmentation and recognition }\end{array}$ \\
\hline 2 & $\begin{array}{c}\text { Chandranath Adak (Griffith University, } \\
\text { Australia / segmentation }\end{array}$ \\
\hline & $\begin{array}{c}\text { Chandranath Adak (Griffith University, } \\
\text { Australia)\& Bhagesh Seraogi (ISI, Kolkata, } \\
\text { India) / recognition }\end{array}$ \\
\hline
\end{tabular}

The Multi-Angle Sclera Database (MASD version 1) used in SSBC 2015 is employed in this competition for the segmentation task [8].

A graphical application was developed using Matlab 7 in the Windows 7 Operating System environment to generate manual segmented masks or ground truths of these sclera images in the dataset, in order to obtain a baseline to evaluate the automatic segmentation algorithms. For the recognition task, segmented sclera eye images were developed by masking the eye images with their respective manual segmented masks (the sclera region is visible and the rest of the eye image is masked).

A set of images at different angles, their manual segmented mask and the masked eye with manual segmented masks are shown in figure 1.

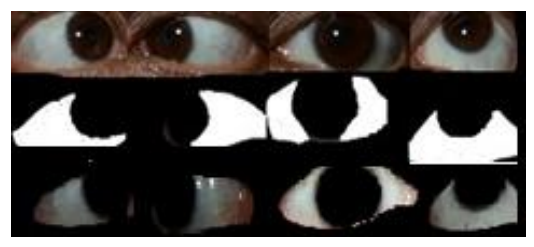

Figure 1: A set of images with different angles, their manual and masked eye with manual segmented masks

For algorithm development purposes, a subset of the database, both eye images and ground truths (1 image for each angle of the first 30 individuals i.e. 120) were provided to the registered participants " of the competition. The participants were asked to provide a Matlab script file (segmentation.m or segmentation.p) that can read the images from a directory and that writes the segmented mask in a particular directory with a naming convention. For the benefit of evaluating the algorithm, and to retain the realtime property of the submitted algorithm, the participants were asked to submit an algorithm which does not take more than 10 seconds to segment and generate a mask for an image on an Intel core i7 processor.

For the recognition task, 32 images (i.e. 8 images for each angle of the first 30) were provided. The participants were required to provide a Matlab script file (training.m or training.p) that can read the images from a directory and generate the training model. Another separate Matlab script file (testing.m or test for training.p) that can read images from a directory prompts which class it belongs to.

The evaluation of the automatic segmented mask with respect to the manual segmented mask is a pixel level binary classification, so a precision and recall measure is employed. Furthermore, recall is considered the measure for ranking the algorithms if the rank is the same for any scenario with respect to precision of the algorithm. The mathematical representation of the precision and recall for our scenario is shown in the following equations.

$$
\text { Precision in } \%=\frac{\text { NPAM }}{\text { NPRS }}
$$




$$
\text { Recall in } \%=\frac{\text { NPAM }}{\text { NRMS }}
$$

Where,

NPAM $=$ Number of pixels retrieved in the sclera region by the automatic segmented mask

NPRS =Number of pixels retrieved in the automatic segmented mask.

NRMS $=$ Number of pixels in the sclera region in the manual segmented mask

For the recognition task, the recognition accuracy was considered for the performance measure.

$$
\text { Accuracy in } \%=\frac{\mathrm{NCRS}}{\mathrm{NS}} \text {. }
$$

Where,

NCRS $=$ Number of Correctly Recognized Samples NS $=$ Number of Samples.

\section{Brief description of the submitted algorithm}

The three segmentation and two recognition algorithms submitted by the three participants are described in this section; they are organized into the next five sub-sections.

\subsection{Segmentation Algorithm by participating team 1}

The participants proposed a new robust segmentation method based on Fuzzy C Means. The technique of fuzzy clustering has become very important in the application of image segmentation. This is due to the large role of uncertainty and imprecision in the images. When the input data contains noise and outliers, the standard Fuzzy cmeans (FCM) fails to give good results. The pixels on an image are highly correlated, i.e. the pixels in the immediate neighborhood possess nearly the same feature data. Therefore, the spatial relationship of neighboring pixels is an important characteristic to aid image segmentation.

A conventional FCM algorithm does not fully utilize spatial information. The spatial FCM algorithm incorporates spatial information and the membership weighting of each cluster is altered after the cluster distribution in the neighborhood is considered. However, the Spatial FCM (SFCM) algorithm is not suitable for revealing non-Euclidean structures of the input data due to the use of Euclidean distances. Thus to overcome this problem, the participants proposed a new Robust Spatial Kernel FCM (RSKFCM) method proposed in [2]. The proposed method considers spatial information and uses the Gaussian kernel function to calculate the distance between the centre of the cluster and the data points. Figure 2 shows the block diagram of the proposed method for Sclera segmentation. Chuang et al. [1] fuzzy c-means algorithm that incorporates spatial information into membership functions for clustering is used for sclera segmentation.

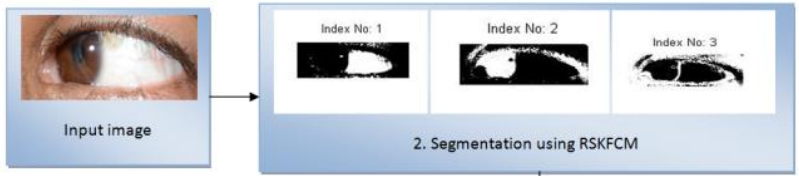

Figure 2: Block diagram of the proposed method

3.2. $1^{\text {st }}$ Segmentation Algorithm by participating team 2 Since the eye image dataset provided for the competition is in color, the participants intended to tackle the problem as a color segmentation problem. But the difficulty arose when the eye-brow and iris color became almost the same. So, instead of seeing it only as a color segmentation problem, the participants tried to find the larger white portion, i.e. the sclera.

The contributor has used an unsupervised technique, so that prior knowledge is not required. Two variations of this technique have been submitted for this competition. In the very first stage, to reduce computation cost, the method converts the color image into gray levels. Then all the peaks are found from the gray-level histogram. Fuzzy C-Means (FCM) clustering is used for segmenting the gray image. Here the number of clusters (C-value) is perceived as the number of peaks ( \pm low threshold $\mathrm{T}$ ) of that histogram. Since the target is to find the sclera, i.e. larger white region, the cluster having the highest gray-value is extracted as the region of interest and the remaining portions turned into black. It is observed that the sclera does not contain any holes, so all the holes are filled in and very small components are filtered out as noise.

\section{3. $2^{\text {nd }}$ Segmentation Algorithm participating team 2}

A second version of the previous algorithm was employed in this work. In this variation, instead of taking only the cluster with the highest gray-value, two clusters having the highest and next-to-highest gray values are chosen. Now on these two clusters, Otsu's binarization is performed. Similar to the above approach, here also hole-filling and noise (small isolated components) removal are performed to obtain a better outcome.

\subsection{Recognition Algorithms from participating team 1} This team proposed a new k-Nearest Neighbor (k-NN) based Sclera Recognition system. The proposed system consists of two steps: Feature Extraction and Matching. The Feature Extraction approach uses the Histogram of Oriented Gradient (HOG) descriptor to extract the features. HOG are feature descriptors used in computer vision and image processing for the purpose of object detection. The technique counts the occurrence of gradient orientations in the localized portion of an input image. The proposed method adopts K-Nearest Neighbor (k-NN) as a pattern classification technique. Nearest Neighbor classifiers are based on learning by analogy, that is, by comparing a given test tuple with training tuples which are similar to it. The 
training tuples are described by $\mathrm{n}$ attributes. Each tuple represents a point in an n-dimensional space. In this way, all of the training tuples are stored in an n-dimensional pattern space. When given an unknown tuple, a k-nearest neighbor $(\mathrm{k}-\mathrm{NN})$ classifier searches the pattern space for the $\mathrm{k}$ training tuples; that which is closest to the unknown tuple is assigned.

\subsection{Recognition Algorithms from participating team 3}

This team has used a multiclass feature-based classifier for sclera recognition. Two types of features have been employed for classification.

The well-known Gabor features are extracted. Empirically, the team has set the no. of scales $(u=5)$, no. of orientations $(\mathrm{v}=8)$, no. of rows $(\mathrm{m}=39)$ and columns $(\mathrm{n}=39)$ of a 2-D Gabor filter [9]. The feature vector is also downsampled to a size of 720 .

On the sclera, the vein-structure holds significant individual information. Since the Canny edge detector [10] uses a Gaussian distribution, it produces a reasonably good amount of change information in the reddish vein color and the white sclera. For this purpose, the team has used such edge detection algorithms and has found the 3 types of density distributions over the sclera image. So, a densitybased feature vector of size 3 has also been employed. The proposed method adopts the k-Nearest Neighbor (k-NN) as a pattern classification technique.

\section{Discussion and results}

In this section we summarized and analyzed the results achieved after applying the submitted algorithms on our MASD version 1 dataset for segmentation and recognition tasks.

\subsection{Sclera segmentation results and discussion}

We maintained the protocol for submissions of the algorithm and then evaluated them by a common framework and ranked them to maintain a fair and unbiased competition among the participants. Through this publication, the participants can assess the performance of their methods relative to the others, and we believed that the competition benefited from this synergy between participants. The results were obtained on the test dataset comprised of 2624 images. In table 3, the final quantitative results are presented for the three algorithms in the competition.

As far as our competition protocol was concerned, we ranked the results by the recall and further ranked by precision for any duplicated ranks generated.

Table 3. Final results of the participants (in \%).

\begin{tabular}{|c|c|c|c|}
\hline Rank & Participating teams & Precision in $\%$ & Recall in $\%$ \\
\hline \hline 1 & 1 & 85.21 & 80.21 \\
\hline 2 & 2 & 75.09 & 70.10 \\
\hline 3 & 2 & 74.01 & 73.20 \\
\hline
\end{tabular}

From the above table it is clear that the results obtained from the algorithm of each participant have reached appreciable figures. Among them, the algorithm of Participant 1 has produced the best results in both the scenarios of precision and recall. The precision and the recall value of the $1^{\text {st }}$ and the $2^{\text {nd }}$ rank also have a large percentage of difference with the second ranking algorithm.

The results obtained, by most of the participants, were appreciable with regards to the statistical measure we employed here. For critical analysis of these impressive results (from the three algorithms), we decided to analyze the characteristics of our images and tested the robustness of the methods to a more critical extent. We undertook to investigate the images where the algorithm failed to produce very good segmented masks. Manipulation of the segmentation of the images with respect to their illumination and lighting conditions was considered in this analysis. Although in this context we must state that the discussion may be considered invidious, since the algorithms were not trained for this type of analysis.

Some intuitive critical research analysis was performed and an attempt was made to find the images in the dataset having a high degree of variation in illumination, to realize the potential of the algorithm with respect to the difference in illumination.

We scrutinized the segmented masks created by the algorithm and found mainly four scenarios where the algorithms failed to successfully obtain a fair mask. The following are four such scenarios:

1) Variation in illumination for one part of the eye $-3(a)$.

2) Variation in illumination at the part of sclera and the other parts of the eye -3 (b and c).

3) Variation in illumination for the total eye image - $3(\mathrm{~d}$ and e).

4) Variation in illumination due to the light source - 3(f \& g).

We consider this problem as a very major problem with regards to the robust segmentation of the sclera because the light source is required to illuminate the eye in order to capture the sclera vessel patterns. Moreover, this lighting or illumination source may also vary with respect to the acquiring conditions. Therefore, it is one of the biggest hindrances of these algorithms to robustly sclera segmentation. Example eye images of these four scenarios and segmented masks created by these algorithms are provided in figure 3 . The basic faults that appear on the segmented mask due to these conditions are:

1) For the first scenario, the lighter part of the sclera is segmented correctly but the darker part is not segmented. Also some parts of the skin were misclassified as the sclera region, mainly the lighter part.

2) For the second scenario, some parts of the sclera mainly 
the vessels patterns, were misclassified.

3) For the third scenario the lighter part of the sclera is segmented correctly, but the darker part is not segmented.

4) For the third scenario in the darker images, for the images where less illumination was applied, the skin region around the eye was misclassified.
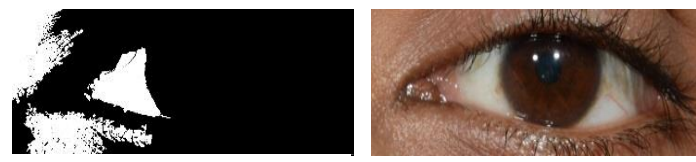

(a)
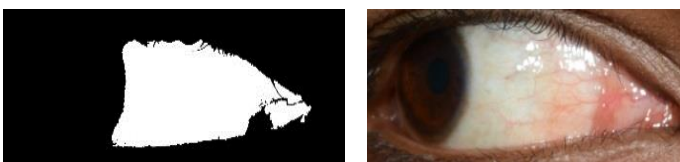

(b)
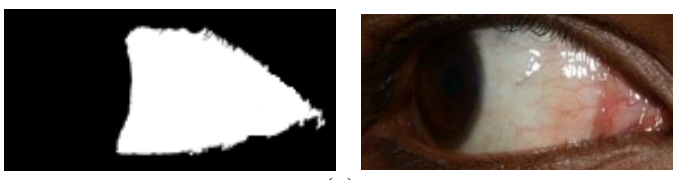

(c)
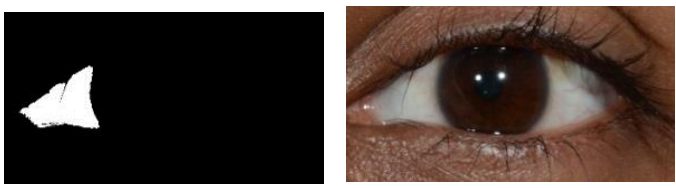

(d)
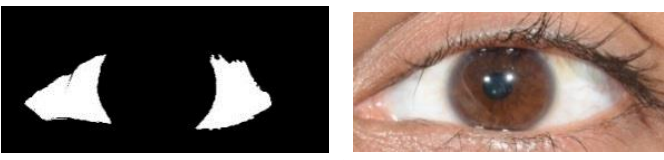

(e)
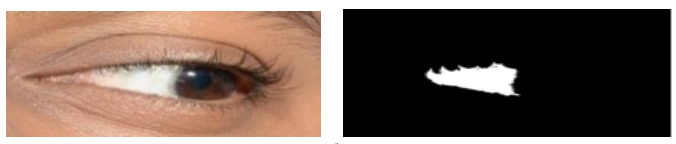

(f)
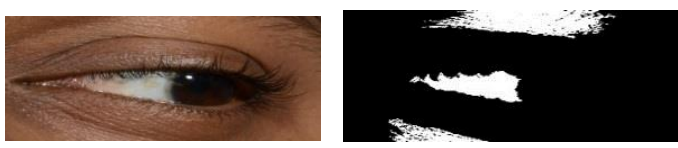

(g)

Figure 3: Effect of segmentation on variation in illumination (a) one part of the eye, $(b \& c)$ the parts of sclera and the other parts of the eye, (d \& e) total eye image and ( $\&$ \& $\mathrm{g}$ ) light source.

We assume the major reason behind this misclassification is the basis of the algorithms. All these algorithms are mainly based analyzing and optimizing on the differences in the color value of the chromatic level of the foreground and the background.
Therefore, an abrupt change significantly affected the algorithms. This analysis has demonstrated the nonfeasibility of a real-life application of these algorithms. However, this kind of analysis requires prior knowledge and the ease of access to the specific scenario.

For further analysis we observed the segmented mask produced by the best algorithm of SSBC 2015 [8]. From the detailed experiment analysis, we found that in that algorithm, details about these changes were also needed for incorporation in the algorithm during training. Moreover, that algorithm (reported in [8] performed substantially better than these algorithms from SSRBC 2016). In contrast i.e. in a controlled learning scenario, that algorithm also failed to pass this robustness.

Therefore the above-mentioned challenge is an open research area in sclera segmentation. To avoid some misclassifications, pre-processing of the eye images could help (i.e. avoiding scenarios such as the introduction of sclera vessel patterns in the masked cluster, etc.). Furthermore, aggregation of the supervised algorithm in [8] and the unsupervised algorithm proposed in SSRBC 2016 could also be a solution to mitigating these challenges.

\subsection{Sclera recognition results and discussion}

Recognition result evaluation was performed on the enhanced sclera images to maintain a fair and unbiased competition among the participants.

Adaptive histogram equalization was performed with a window size of $42 \times 42$ on the green channel of the masked sclera images to make the vessel structure more prominent. An adaptive histogram at a clip limit of 0.01 , with a full range and distribution exponential is used to get the best result.

Furthermore, the Discrete Meyer wavelet was used to enhance the vessel patterns. Low pass reconstruction with a cut off range of -0.00009 and a window size of $3 \times 3$ was employed.

Next, another round of histogram equalization was performed with the same parameters above. An enhanced sclera vessel image and one masked with its manual segmented mask is shown in figure 4.
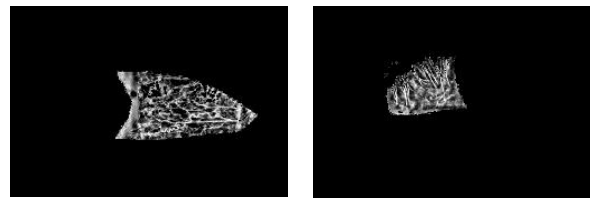

Figure 4: An enhanced sclera vessel image and one masked with its manual segmented mask.

For training and testing we divided the dataset irrespective of the gaze angle. Eight images from four different gaze angles were used for training and the remaining eight for testing. In Table 4 the final quantitative results are 
presented for the two recognition algorithms in the competition. As far as our competition protocol was concerned, we undertook the ranking by the accuracy percentage achieved by the algorithms.

Table 4. Final recognition results for each participants

\begin{tabular}{|c|c|c|}
\hline Rank & Participating teams & Accuracy in \% \\
\hline \hline 1 & 3 & 82.11 \\
\hline 2 & 1 & 80.55 \\
\hline
\end{tabular}

It can be easily concluded from the results in the table 4 that the algorithms are invariant to the sclera gaze angle. It is also clear from table 4 that the algorithms of participants 1 have performed better. Both algorithms proposed by these participants used either texture or shape feature or both. The sclera vessel patterns are enriched with both the shapebased feature and the texture feature. Therefore as participants 3 used a blended feature of both the shapebased and texture-based features, they achieved higher recognition accuracy than participants 1 .

In this context it is also worth mentioning that the sclera patterns are very rich in both local and global features, therefore to achieve higher accuracy it could be useful to blend both the local and the global features. To analyze this usefulness of blending effect we used the feature used in [11] on the dataset used for the recognition task. The patched-based LDP (Local Directional Pattern), a local descriptor that extracts the local features followed by the Spatial Pyramid Matching, which takes care of the global features, have significantly achieved higher recognition accuracy than the algorithms proposed by the two participants (using k-NN pattern classification techniques).

It can also be observed that the algorithm used K-NNs as the classifier; therefore a stronger classifier such as SVMs (Support Vector Machines) could enhance the recognition accuracy. To analysis this, we have also conducted experiments with the patch-based LDP in a combination with SVMs as the classifier. The experiments achieved higher recognition accuracy than the scenario when K-NNs were used with a patched-based LDP. Therefore it is quite clear from the above analysis that the blending of features and the use of a stronger classifier can help to achieve higher recognition accuracies in the sclera recognition task.

\section{Conclusions and future Scope}

The $1^{\text {st }}$ Sclera Segmentation and Recognition Benchmarking Competition, SSRBC 2016 was organized with the primary goals to record the recent advancements in sclera segmentation and recognition techniques. Moreover it also aims to provide a common platform to evaluate sclera segmentation and recognition algorithms using a unique multi-angle sclera dataset. Subsequently, the showcasing of the competition in one of the most recognized gatherings in the biometric community i.e. ICB 2016 and promoting them via different electronic media, have also increased the interest of researchers using this particular biometric trait. Furthermore, the conceived competition has satisfactorily fulfilled all of the above aims and the popularity and the interest of the participants were noteworthy. In addition the algorithms submitted by the participants demonstrate appreciable results on our proposed dataset. The critical analysis undertaken on the results of the different algorithms will also provide a way forward for further research. One very important aspect of the research is the availability of datasets publicly, which is enriched with a wider variety of multi-angle or eye gaze scenarios. In addition, to the best of our knowledge, no such datasets are publicly available, and the availability of this proposed dataset will fill that gap. The successful organization and the appreciating impact of this competition have inspired the organizers to plan further competitions on the sclera biometrics paradigm in the near future in conjunction with BTAS 2016, namely the $3^{\text {rd }}$ Sclera Segmentation Benchmarking Competition, SSBC 2016.

\section{References}

[1] N. Otsu, A threshold selection method from gray-level histograms, IEEE Trans. on Systems, Man and Cybernetics, vol.9, no.1, 62-66, 1979.

[2] K.S Chuang, H.L Hzeng, S. Chen, J. Wu and T.J Chen ,Fuzzy c-means clustering with spatial information for image segmentation", Computerized Medical Imaging and Graphics, vol. $30,9-15,2006$

[3] A. Das, U. Pal, M. A. Ferrer and M. Blumenstein, A New Method for Sclera Vessel Recognition using OLBP, Chinese Conference on Biometric Recognition ,LNCS 8232: 370-377, 2013.

[4] A. Das, U. Pal, M. A. Ferrer and M. Blumenstein, Sclera Recognition Using D-SIFT, In 13th International Conference on Intelligent Systems Design and Applications: 74-79, 2013.

[5] A. Das, U. Pal, M. Blumenstein and M. A. Ferrer, Sclera Recognition - A Survey, Advancement in Computer Vision and Pattern Recognition: 917 -921, 2013.

[6] A. Das, U. Pal, M. A. Ferrer and M. Blumenstein, Fuzzy Logic Based Sclera Recognition, In FUZZ-IEEE: 561-568, 2014.

[7] A. Das, U. Pal, M. A. Ferrer and M. Blumenstein, Multi-angle Based Lively Sclera Biometrics at a Distance, IEEE Symposium Series on Computational Intelligence, 22-29, 2014.

[8]A. Das, U. Pal, M. A. Ferrer and M. Blumenstein, SSBC 2015: Sclera Segmentation Benchmarking Competition, BTAS 2015, 16, 2015.

[9] M. Haghighat, S. Zonouz, and M. Abdel-Mottaleb, CloudID: Trustworthy cloud-based and cross-enterprise biometric identification, Expert Systems with Applications, vol. 42, no. 21, 7905-7916, 2015.

[10] J. Canny, A Computational Approach to Edge Detection, IEEE Trans. on PAMI, vol. 8, no.6,679-698, 1986.

[11] A. Das, U. Pal, M. F. Ballester and M. Blumenstein, A New Efficient and Adaptive Sclera Recognition System, IEEE Symposium Series on Computational Intelligence, December 912,2014 